\title{
Hydrodynamic Fluctuations and Instabilities in Ordered Suspensions of Self-Propelled Particles
}

\author{
R. Aditi Simha* and Sriram Ramaswamy\# \\ Centre for Condensed-Matter Theory, Department of Physics, Indian Institute of Science, Bangalore 560 012, India
}

\begin{abstract}
We construct the hydrodynamic equations for suspensions of self-propelled particles (SPPs) with spontaneous orientational order, and make a number of striking, testable predictions: (i) Nematic SPP suspensions are always absolutely unstable at long wavelengths. (ii) SPP suspensions support novel propagating modes at long wavelengths, coupling orientation, flow, and concentration. (iii) In a wave number regime accessible only in low Reynolds number systems such as bacteria, polar-ordered suspensions are invariably convectively unstable. (iv) The variance in the number $N$ of particles, divided by the mean $\langle N\rangle$, diverges as $\langle N\rangle^{2 / 3}$ in polar-ordered SPP suspensions.
\end{abstract}

Fish, birds, and swimming cells [1-3] are frequently found to move coherently in large groups [4-9] through the fluid medium they inhabit. Can general principles, like those used so successfully for ordered phases at equilibrium [10], reveal the laws governing the long-wavelength dynamics and fluctuations of these striking and ubiquitous examples of liquid-crystalline [11] order in suspensions of self-propelled particles (SPPs)? Although we know of no physics experiments on ordered SPP suspensions, Gruler [12] has studied ordered phases of living cells on a solid substrate, and finds "living liquid-crystalline" [13] phases corresponding to two distinct types of cells: apolar, that is, elongated but head-tail symmetric, and polar, distinguishing front from rear. The ordered phases formed by polar SPPs have a nonzero macroscopic drift velocity $v_{0}$. Those formed by apolar SPPs have a macroscopic axis $\hat{\mathbf{n}}$ of orientation but, like true nematics [14], do not distinguish $\hat{\mathbf{n}}$ from $-\hat{\mathbf{n}} ;$ they thus have $\boldsymbol{v}_{0}=0$. Migratory cells such as white blood cells are polar in this sense, while melanocytes, which distribute pigment in the skin, are apolar [12]. Although an isolated melanocyte is incapable of directed motion, we still term it an SPP because it displays spontaneous, self-generated energy-dissipating activity, in the form of a pair of symmetrically pulsating dendrites [12]. The pioneering studies of [5] and $[6,8,9]$ consider ordered flocks drifting through a passive frictional background. In [7] as well, fluid flow is ignored in the analysis, although viscosity is mentioned as the source of damping. This is appropriate for polar SPPs on a substrate as in [12], but not for SPPs immersed in a bulk fluid. In this Letter we use symmetry and conservation laws to construct the complete equations of motion for small, long-wavelength disturbances in polar as well as apolar ordered SPP suspensions, including the flow of the ambient fluid.

Our main results, which we now summarize, contain striking, experimentally testable signatures of the nonequilibrium nature of ordered SPP suspensions, and of the crucial role of the hydrodynamics of the ambient fluid medium: Purely nematic order in SPP suspensions is always destabilized at small enough wave number $q$, by a coupled splay of the axis of orientation and a correspond- ing Taylor-Couette-like circulation of the velocity field, oriented near $45^{\circ}$ to the nematic axis, with a growth rate linear in $q$.

Polar ordered suspensions display some rather original propagating modes as a result of the interplay of hydrodynamic flow with fluctuations in the ordering direction and the concentration: a pair of bend-twist waves (with no analog in the work of [9]), with wave speeds; see Fig. 1 and Eq. (7), and three waves which are a combination of splay, concentration, and drift (a generalization of those in [9]), whose speeds are better understood from Fig. 2 than from an equation.

The results in Figs. 1 and 2 and Eq. (7) are obtained when viscous damping is neglected, which is valid for wave numbers $q \ll v_{0} / \nu$ where $\nu$ is a typical kinematic viscosity. Experiments on bacterial suspensions are, however, likely to be in the $\operatorname{Re} \ll q a \ll 1$ regime, where $\operatorname{Re}=$ $v_{0} a / \nu$ is the Reynolds number of an SPP of size $a$. In that (Stokesian) limit we find, remarkably, that a polar-ordered suspension is always unstable for wave vectors $\mathbf{q}$ near $45^{\circ}$ to the nematic axis, with a growth rate $\sim v_{0} / a$, independent of $|\mathbf{q}|$. The instability is "convective": it travels with a speed $\sim \boldsymbol{v}_{0}$ as it grows.

Last, number fluctuations in polar-ordered SPP suspensions are anomalously large. The variance $\left\langle(\delta N)^{2}\right\rangle$ in the number of particles, scaled by the mean $N$, is predicted to diverge as $N^{2 / 3}$.

The results above follow from a set of hydrodynamic equations of motion, which we now construct by generalizing [10]. Assume we have nematic or polar-ordered phases of SPPs, with orientation given by a unit director field $\hat{\mathbf{n}}$ aligned on average in the $z$ direction. The nematic is invariant under $\hat{\mathbf{n}} \rightarrow-\hat{\mathbf{n}}$; the polar-ordered state is not. The slow variables [10] are (a) the "conserved modes" which, for an incompressible suspension, are [15] the fluctuations $\delta c(\mathbf{r}, t)$ at point $\mathbf{r}$ and time $t$ in the local concentration $c$ of suspended particles about its mean $c_{0}$ and the total (solute + solvent) momentum density $\mathbf{g}(\mathbf{r}, t) \equiv$ $\rho \mathbf{u}(\mathbf{r}, t)$, where $\rho$ is the constant mass density of the suspension and $\mathbf{u}$ the hydrodynamic velocity field; and (b) the "broken-symmetry" variables which, for both 


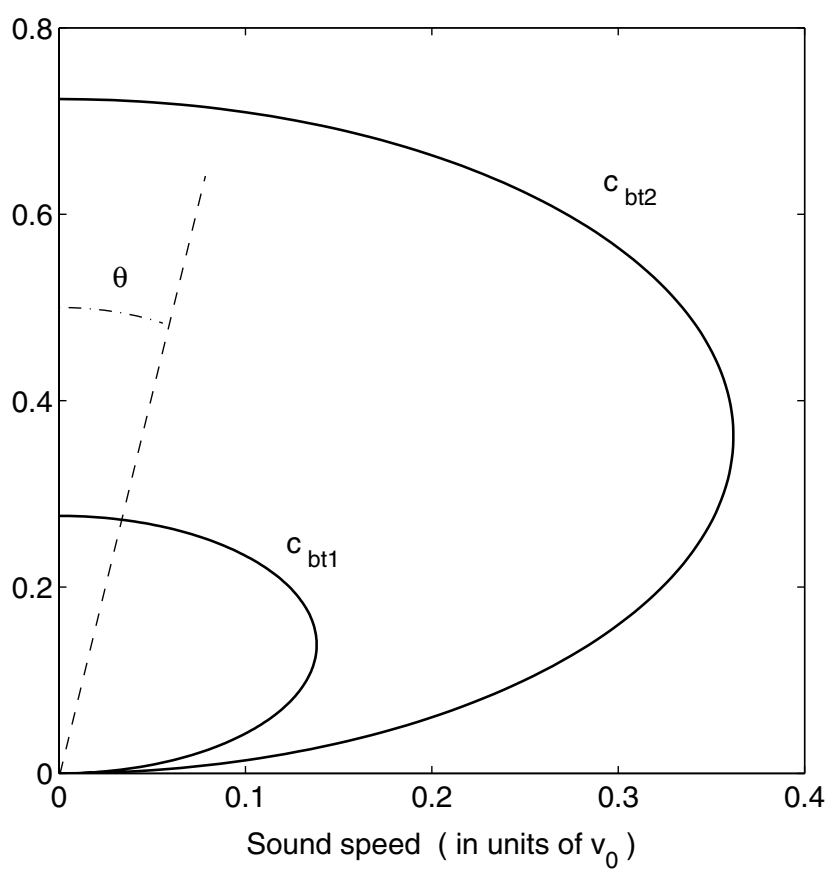

FIG. 1. A polar plot of speed of the bend-twist modes as a function of the angle $\theta$ between the propagation direction and the $z$ axis.

polar and nematic phases, are simply the deviations $\mathbf{\delta} \mathbf{n}_{\perp}=$ $\hat{\mathbf{n}}-\hat{\mathbf{z}}$. In polar-ordered suspensions, the drift velocity of SPPs relative to the fluid is $v_{0} \hat{\mathbf{n}}$. Since these are driven systems, the forces entering the equations of motion need not arise from a free-energy functional, and any term not explicitly forbidden by symmetry or conservation laws must be included. Moreover, there are a priori no relations among the phenomenological parameters other than those

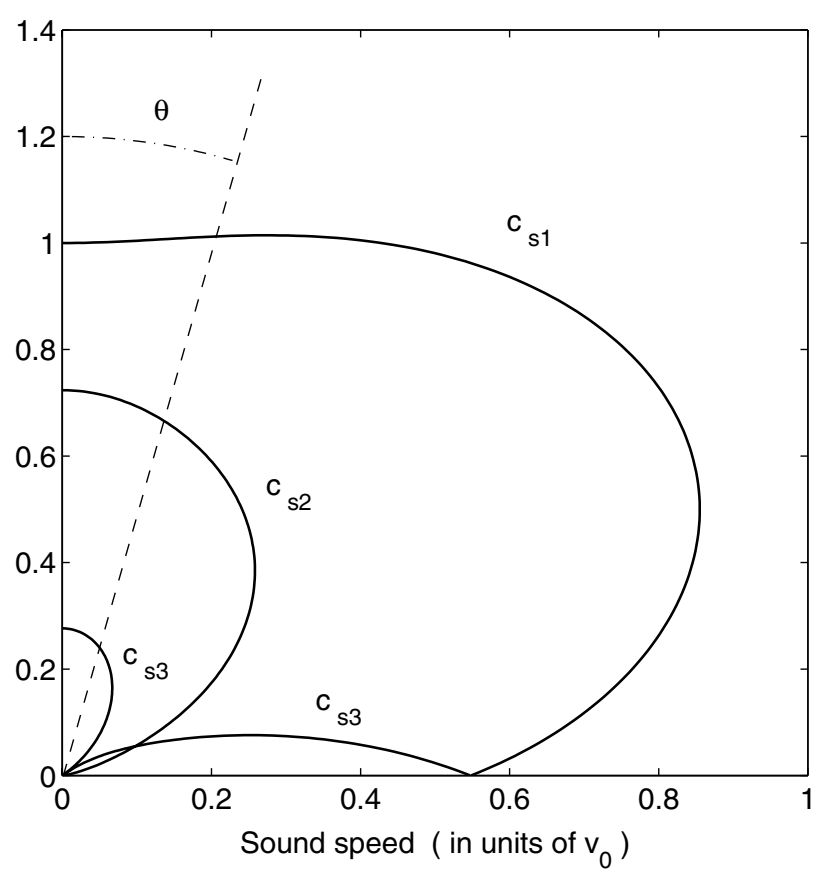

FIG. 2. A polar plot of the speed of the splay-concentration and drift waves as a function of the angle $\theta$ between the propagation direction and the $z$ axis.

required by the geometrical symmetries of the problem. Note that our coarse-grained description applies on length scales $\gg a$, the particle size.

We now present the equations of motion which follow from these arguments, and explain the physical origin of the terms therein. We begin with the polar-ordered case. The equations for the nematic case follow by dropping terms which violate $z \rightarrow-z$ symmetry. The director field obeys

$$
\partial_{t} \delta \mathbf{n}_{\perp}=-\lambda_{1} v_{0} \partial_{z} \delta \mathbf{n}_{\perp}-\sigma_{1} \nabla_{\perp} \delta c+\frac{1}{2}\left(\partial_{z} \mathbf{u}_{\perp}-\nabla_{\perp} u_{z}\right)+\frac{1}{2} \gamma_{2}\left(\partial_{z} \mathbf{u}_{\perp}+\nabla_{\perp} u_{z}\right)+\ldots
$$

The first term on the right-hand side of (1) represents advection of distortions by the mean drift $v_{0}$ [16]. The second says simply that SPPs move from high to low concentration-a nonequilibrium "osmotic pressure." These terms are a consequence of the lack of $z \rightarrow-z$ symmetry, and are present in the model of $[6,8,9]$ as well. The third term on the right says that in the absence of other forces the director rotates at the same rate as the fluid. Together with the fourth term, containing the phenomenological parameter $\gamma_{2}$, this gives rise to the "flowalignment" [14,17] phenomenon, well-known in nematic hydrodynamics. These two terms are, of course, absent in $[6,8,9]$ and are central to our work. The ellipsis in (1) subsumes diffusive terms of higher order in gradients, as well as nonlinear terms.

The equation of motion for $\mathbf{g}$ has the form $\partial_{t} g_{i}=$ $-\nabla_{j} \sigma_{i j}$, as in any momentum-conserving system, where $\sigma_{i j}$ is the stress tensor. What distinguishes SPP systems is a contribution $\sigma_{i j}^{(p)} \propto n_{i} n_{j}-(1 / 3) \delta_{i j}$ to the shear stress, as a result of the self-propelling activity. We must allow for such a term because no symmetry can rule it out in these driven systems [18]. To derive $\sigma_{i j}^{(p)}$ from the dynamics of individual SPPs note that, by Newton's Third Law, the forces exerted by an SPP on the fluid and by the fluid on an SPP are equal and opposite; i.e., the combined system of SPPs + fluid is force-free. Thus, in the equation of motion for the total momentum density $\mathbf{g}$, the force density associated with each SPP and the ambient fluid must integrate to zero. Averaging over the detailed swimming movements, it follows that the simplest model for, say, the $\alpha$ th SPP is a rod with axis $\hat{\mathbf{n}}_{\alpha}(t)$, at time $t$, with point forces of equal magnitude $f$ on its ends, directed along $\pm \hat{\mathbf{n}}_{\alpha}$ [19]. For a collection of SPPs with centers at $\mathbf{r}_{\alpha}(t)$ and ends at $\mathbf{r}_{\alpha}+a \hat{\mathbf{n}}_{\alpha}$ and $\mathbf{r}_{\alpha}-a^{\prime} \hat{\mathbf{n}}_{\alpha}$, where we have allowed for force centers asymmetrically disposed about the center of the SPP, this yields a force density (divergence of stress) 


$$
\begin{aligned}
-\nabla \cdot \sigma^{(p)} \equiv \mathbf{f}^{(p)}(\mathbf{r}, t) & \\
=f \sum_{\alpha} \hat{\mathbf{n}}_{\alpha}(t) & \left\{\delta \left(\left[\mathbf{r}-\mathbf{r}_{\alpha}(t)-a \hat{\mathbf{n}}_{\alpha}(t)\right]\right.\right. \\
& \left.-\delta\left[\mathbf{r}-\mathbf{r}_{\alpha}(t)+a^{\prime} \hat{\mathbf{n}}_{\alpha}(t)\right]\right\} .
\end{aligned}
$$

The apolar and polar SPPs we mentioned early in this Letter correspond, respectively, to $a=a^{\prime}$ and $a \neq a^{\prime}$. Expanding the delta functions in (2) about $\mathbf{r}_{\alpha}$ gives

$$
f_{i}^{(p)} \simeq-\frac{a+a^{\prime}}{2} f \nabla_{j} \sum_{\alpha} \hat{n}_{\alpha i} \hat{n}_{\alpha j} \delta\left(\mathbf{r}-\mathbf{r}_{\alpha}\right)+O(\nabla \nabla) .
$$

In a coarse-grained description this clearly leads to a shear stress

$$
\sigma_{i j}^{(p)}(\mathbf{r}, t)=\frac{a+a^{\prime}}{2} f c(\mathbf{r}, t)\left(n_{i} n_{j}-\frac{1}{3} \delta_{i j}\right)+O(\nabla)
$$

as claimed above. Note that only the sum $a+a^{\prime}$ appears in (4); to leading order in gradients, the self-propelling stress is the same for polar and apolar phases. Using this stress in the momentum equation $\partial_{t} g_{i}=-\nabla_{j} \sigma_{i j}$, Fourier transforming in space, replacing $\mathbf{g}$ by the hydrodynamic velocity field $\mathbf{u}=\mathbf{g} / \rho$, and projecting transverse to the wave vector $\mathbf{q}$ to impose incompressibility $(\nabla \cdot \mathbf{u}=0)$ yields, after some algebra,

$$
\frac{\partial \mathbf{u}_{\perp}}{\partial t}=-i w_{0} q_{z}\left(\mathbf{I}-2 \frac{\mathbf{q}_{\perp} \mathbf{q}_{\perp}}{q^{2}}\right) \boldsymbol{\delta} \mathbf{n}_{\perp}-i \frac{q_{z}^{2}}{q^{2}} \alpha\left(\mathbf{q}_{\perp} \delta c\right)+\ldots
$$

where $\mathbf{I}$ is the unit tensor, and $\alpha \sim f a / \rho$ and $w_{0} \sim c_{0} \alpha$ are phenomenological constants proportional to the activity of the SPPs. In (5), nematic-elastic torques [14] and viscous forces, both of which are subdominant at small $q$, have been ignored, as have inertial and other nonlinearities. The pressurelike $\alpha$ term despite transverse projection and the acceleration proportional to the bend $q_{z} \delta \mathbf{n}_{\perp}$ arise purely because of self-propulsion [18].

Number conservation of SPPs implies $\partial_{t} \delta c=-\nabla \cdot \mathbf{j}$, where the current $\mathbf{j}=c \boldsymbol{v}_{0} \hat{\mathbf{n}}$ apart from advection by fluid flow and diffusion. To lowest order in gradients and fields, this tells us that

$$
\left(\partial_{t}+v_{0} \partial_{z}\right) \delta c+c_{0} v_{0} \nabla_{\perp} \cdot \delta \mathbf{n}_{\perp}
$$

which says the concentration changes by advection by the mean drift $v_{0}$ or by local splay (since that implies a divergence in the local SPP velocity).

We can now extract the propagating waves and instabilities mentioned at the start of this paper, from the dispersion relation, frequency $\omega$ as a function of wave vector $\mathbf{q}$, for modes varying as $\exp (i \mathbf{q} \cdot \mathbf{r}-i \omega t)$, implied by Eqs. (1), (5), and (6).

Bend-twist waves: Taking the curl of (1) and (5) yields the coupled dynamics of bend or twist $\left(\nabla \times \mathbf{n}_{\perp}\right)$ and vorticity $\left(\nabla \times \mathbf{u}_{\perp}\right)$. Fourier transforming in space and time yields $\omega=c_{b t}(\theta) q$, with wave speeds (see Fig. 1)

$$
c_{b t}(\theta)=\left(c_{1} \pm c_{2}\right) \cos \theta,
$$

where $\theta$ is the angle between the propagation vector and the ordering direction, and $c_{1}$ and $c_{2}$ (of order the drift speed $v_{0}$ of the SPPs) are phenomenological constants. An analysis ignoring hydrodynamic flow, as in $[6,8,9]$, would instead predict purely diffusive relaxation for bend and twist.

Splay-concentration and drift waves: Taking the divergence of (1) and (5) results in coupled equations of motion for $\nabla \cdot \mathbf{n}_{\perp}, \nabla \cdot \mathbf{u}_{\perp}$, and $\delta c$. The resulting wavelike eigenmodes are a generalization of those seen in [9]. Their speeds obey a messy cubic equation which, we can show [20], has real solutions for a finite range of parameter values. The speeds as functions of direction, for typical parameter values, are illustrated in Fig. 2.

Viscous effects, and the instability of polar SPP suspensions in the Stokesian limit: So far, we have ignored damping terms in the equations of motion since they enter at next-to-leading order in wave number $q$. There are three types of such terms: (i) viscous damping $\sim \nu q^{2} \mathbf{u}$ in the momentum equation (where $\nu$ is a typical kinematic viscosity of the suspension); (ii) $D q^{2} \delta c$ in the concentration equation, $D$ being a diffusivity; (iii) $D_{n} q^{2} \boldsymbol{\delta} \mathbf{n}_{\perp}$ in the director equation where $D_{n}$ is a director diffusivity which we expect to be comparable to $D$. In bacteria, diffusivities are of order $10^{-6} \mathrm{~cm}^{2} / \mathrm{s} \mathrm{[3]} \mathrm{and} \mathrm{can} \mathrm{clearly} \mathrm{be} \mathrm{neglected}$ relative to the viscosity. In large-Re systems such as fish, we have no reliable estimate of $D$, but it seems reasonable to assume it is no larger than $\nu$. Accordingly, when damping terms are included, the modes will remain propagating only for $q a<v_{0} a / \nu \equiv$ Re, i.e., at practically all length scales in the case of fish, but only at exceedingly large length scales for bacteria, where $\operatorname{Re} \sim 10^{-6}$ to $10^{-4}$. The regime of primary interest for bacteria is $\nu q^{2} \gg v_{0} q$, i.e., $q a \gg v_{0} a / \nu \equiv \operatorname{Re}$, where viscous damping dominates. For such low Reynolds number systems we can use the Stokesian approximation, where the velocity field $\mathbf{u}$ is determined instantaneously by a balance between viscous and other (in this case self-propulsive) stresses. We can thus replace the acceleration in (5) by $\nu q^{2} \mathbf{u}$ and use it to eliminate $\mathbf{u}$ from (1) and (6). This yields effective equations of motion for the splay $\nabla \cdot \boldsymbol{\delta} \mathbf{n}_{\perp}$ and $\delta c$, the coupled dynamics of which can be seen to yield an unstable mode with growth rate

$$
\Gamma(\theta) \sim B\left(\gamma_{2} \cos 2 \theta+1\right) \cos 2 \theta
$$

with $|B| \sim f \phi / a^{2} \eta \sim v_{0} \phi / a, \phi$ being the volume fraction of SPPs. This implies an instability just above or just below $\theta=\pi / 4$, depending on the sign of $B$. The mode has a nonzero real part $\sim \pm v_{0} q \cos \theta$ : this is a convective instability, which is seen if one follows the traveling waves. Note that $\Gamma$ is independent of the magnitude of $\mathbf{q}$, as a result of the long-ranged hydrodynamic interaction in the Stokesian limit. For $q a \gtrsim \phi^{1 / 2}$, director and particle diffusion enter to restabilize the mode, so the unstable regime is $\operatorname{Re} \ll q a \ll \phi^{1 / 2}$.

Instability of SPP nematic suspensions: The dynamics of an SPP nematic suspension can be obtained from (1), (5), and (6) simply by omitting all terms not invariant 
under $z \rightarrow-z$, i.e., by setting $v_{0}=\sigma_{1}=\alpha=0$. This can readily be shown to yield the dispersion, $\omega^{2} \sim$ $-A q^{2} \cos 2 \theta\left(\gamma_{2} \cos 2 \theta+1\right)$, where $|A| \sim w_{0}$. Physically, this can be understood as follows. The nematic symmetry $z \rightarrow-z$ means that a distortion with wave vector at exactly $45^{\circ}$ has no wavelike response: it does not know which way to go, so the $O\left(q^{2}\right)$ contribution to the squared frequency $\omega^{2}(\mathbf{q})$ vanishes at this angle. $\omega^{2}$ must thus change sign as $\theta$ crosses $45^{\circ}$. For $\theta=\pi / 4-\epsilon$, where $\epsilon$ is small and has the same sign as $A$, we thus get an instability with $\omega(\mathbf{q}) \sim$ $i \sqrt{|\epsilon|} q-i q^{2}$. Here the last term appears when diffusive and viscous terms and the conventional nematic-elastic torques are included, and will stabilize modes at larger wave number. For an SPP nematic on a substrate as in [12], a wave-number-independent damping enters the momentum Eq. (5) and in general suppresses this instability [20].

Fluctuations: In striking contrast to systems at thermal equilibrium, ordered suspensions of SPPs display giant number fluctuations. To see this, we must include noise, as well as the damping terms whose form we have discussed, in the equations of motion. For a general SPP suspension one expects noise sources of three kinds: thermal Brownian motion (negligible for particles larger than a few microns), many-particle hydrodynamic interactions, and intrinsic fluctuations in the self-propelling activity of individual SPPs. In our coarse-grained description it is reasonable to assume Gaussian noise sources $\mathbf{f}_{u}, f_{c}$, and $\mathbf{f}_{n}$, delta correlated in time, in the equations of motion for $\mathbf{u}, c$, and $\mathbf{n}$. Momentum and number conservation imply variances $\sim q^{2}$ for $\mathbf{f}_{u}$ and $f_{c}$ for wave number $q \rightarrow 0$, and nonzero for $\mathbf{f}_{n}$. With these noise and damping terms in place we can calculate correlation functions of the various fields in the steady state. We find that the structure factor $S(\mathbf{q})=c_{0}^{-1} \int_{\mathbf{r}} \exp -i \mathbf{q} \cdot \mathbf{r}\langle\delta c(\mathbf{0}) \delta c(\mathbf{r})\rangle \sim 1 / q^{2}$, which implies that the variance of the number of particles, scaled by the mean, grows as $L^{2} \sim N^{2 / 3}$ for a three-dimensional region of linear size $L$ containing $N$ particles on average. Physically, this happens because distortions in the director produce mass flow. Since the director fluctuations-a broken-symmetry mode-are large, this results in giant number fluctuations as well. Similar supernormal number variances were predicted $[6,8,9]$ for SPPs on a substrate; it is remarkable that they survive here in the presence of long-ranged hydrodynamic interactions.

Let us now estimate the numerical values of the effects we predict. All wave speeds are expected to be of order the drift speed of an SPP (from $\mu \mathrm{m} / \mathrm{s}$ to $\mathrm{cm} / \mathrm{s}$ as one goes from bacteria to fish). If we take the kinematic viscosity $\nu$ to be that of water, then the growth rate of the convectively unstable mode in Stokesian polar-ordered SPP suspensions, say, for bacteria (where velocities are $\sim 10 \mu \mathrm{m} / \mathrm{s}$, size $a \sim \mu \mathrm{m}$, and $\phi$ a few percent), is $0.1 \mathrm{~s}^{-1}$. We look forward to experimental tests of our predictions, on living organisms or perhaps on artificial SPP suspensions.

An analysis of the complete (not linearized) equations of motion, now underway, will show how nonlinear fluctuation effects renormalize the speeds and dampings of the modes presented here, and whether they alter the manner in which number fluctuations diverge with size. The full equations of motion will also prove useful in understanding the effect of imposed shear flows on the state of order of SPP suspensions, and the role of boundaries [21] and topological defects in these systems. These, as well as other results on SPP nematics on substrates and an analysis of isotropic SPP suspensions [3], will appear elsewhere [20].

S. R. thanks the Aspen Center for Physics for support while part of this work was done.

*Electronic address: aditi@physics. iisc.ernet.in

\#Electronic address: sriram@physics.iisc.ernet.in

[1] J. Lighthill, SIAM Rev. 18, 161 (1976).

[2] T. J. Pedley and J. O. Kessler, Annu. Rev. Fluid Mech. 24, 313 (1992).

[3] X.-L. Wu and A. Libchaber, Phys. Rev. Lett. 84, 3017 (2000); G. Grégoire, H. Chaté, and Y. Tu, Phys. Rev. E 64, 011902 (2001).

[4] C. Reynolds, Comput. Graph. 21, 25 (1987); J. Deneubourg and S. Goss, Ethol. Ecol. Evolution 1, 29 (1989); A. Huth and C. Wissel, in Biological Motion, edited by W. Alt and E. Hoffmann (Springer-Verlag, New York, 1990); B. L. Partridge, Sci. Am. 246, No. 6, 114 (1982).

[5] T. Vicsek, A. Czirók, E. Ben-Jacob, I. Cohen, and O. Shochet, Phys. Rev. Lett. 75, 1226 (1995).

[6] J. Toner and Y. Tu, Phys. Rev. Lett. 75, 4326 (1995).

[7] N. Shimoyama, K. Sugawara, T. Mizuguchi, Y. Hayakawa, and M. Sano, Phys. Rev. Lett. 76, 3870 (1996).

[8] J. Toner and Y. Tu, Phys. Rev. Lett. 80, 4819 (1998).

[9] J. Toner and Y. Tu, Phys. Rev. E 58, 4828 (1998).

[10] P. C. Martin, O. Parodi, and P. S. Pershan, Phys. Rev. A 6, 2401 (1972); D. Forster, Hydrodynamic Fluctuations, Broken Symmetry, and Correlation Functions (Benjamin, Reading, 1975).

[11] Reference [9] suggests that crystalline, translational order may be seen in flocks as well.

[12] H. Gruler, U. Dewald, and M. Eberhardt, Eur. Phys. J. B 11, 187 (1999); R. Kemkemer, D. Kling, D. Kaufmann, and H. Gruler, Eur. Phys. J. E 1, 215 (2000).

[13] H. Gruler, M. Schienbein, K. Franke, and A. deBoisfleuryChevance, Mol. Cryst. Liq. Cryst. 260, 565 (1995).

[14] P. G. de Gennes and J. Prost, The Physics of Liquid Crystals (Clarendon Press, Oxford, 1995).

[15] We ignore heat diffusion, nutrient uptake, excretion, reproduction, and death.

[16] As in $[6,8,9]$, the speed of advection is not in general equal to the drift speed, hence the factor $\lambda_{1}$.

[17] D. Forster, Phys. Rev. Lett. 32, 1161 (1974).

[18] Such terms are ruled out in equilibrium nematics by the requirement that force densities arise from a free-energy functional via Poisson-bracket relations.

[19] See J.-B. Manneville, P. Bassereau, S. Ramaswamy, and J. Prost, Phys. Rev. E 64, 021908 (2001).

[20] R. A. Simha and S. Ramaswamy (to be published).

[21] Y. Tu, Physica (Amsterdam) 281A, 30-40 (2000). 